\title{
Editorial
}

\section{Making an impact}

I was deeply honoured when asked to write this editorial on the occasion of the coming of the $30^{\text {th }}$ birthday of the journal Integrated Computer-Aided Engineering (ICAE). A birthday is always an occasion to look into the past and as a statistician I cannot help to do this based on numbers and figures. I used the numbers provided by Scimago Journal \& Country Rank at https://www.scimagojr.com/journalsearch.php?q= $18197 \&$ tip $=$ sid.

A journal publishing papers that are not cited anywhere does not have a true impact in the scientific community. I am aware that summarising a complex issue by just one number might be an oversimplification. But, this is what we do every day - especially in statistics. So, I would like to take a closer look at the citations of ICAE papers.

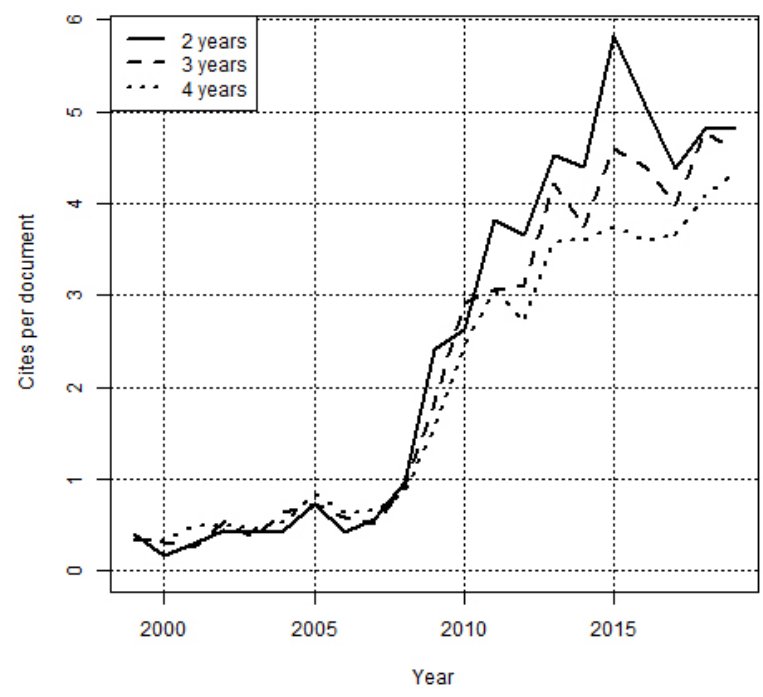

Fig. 1. Average number citations per document.

Figure 1 shows the citations per document over the years. It in the year 2000, each paper was in average cited by less than 0.5 other papers within 2-4 years.
This means that the majority of the papers were not cited at all. And indeed, almost $80 \%$ of the papers published in 2000 in ICAE were not cited at all. This situation is quite common for young journals. It often takes more than a decade until a journal is established properly in the scientific community. After 2007 the citations of ICAE started to boost and reached almost 5 citations per document recently. This increase of citations was not caused by a few extremely popular papers with a high number of citations, but by the majority of papers. In 2019 , more than $80 \%$ of the papers were cited at least once. The number of citation within the last two years - indicated by the solid line in Fig. 1 - is essential for the Impact Factor of a journal. Speaking of the Impact Factor, ICAE has achieved a value of 4.7 for 2020, which is quite remarkable for an engineering journal.

Probably more important than the Impact Factor is the ranking of a journal compared to other journal in related areas. In the year 2000, the still young ICAE was considered to be in the lower quartile in the four fields of Artificial Intelligence, Computational Theory and Mathematics, Computer Science Applications and Theoretical Computer Science, i.e. among all journals in the corresponding fields at least $75 \%$ were considered to be more prestigious. Only for the field of Software, it was in the second quartile, still meaning that more than have of the journals in the field of Software were ranked higher than ICAE. As I mentioned, it often takes more than a decade to establish a journal properly. So in 2020 , ICAE is in all the five above mentioned fields in the first quartile, i.e. it is among the $25 \%$ best journals in these fields.

What or who is the source of this success? I cannot prove it, but I assume that most of this success can be accredited to the Editor-in-Chief, Professor Hojjat Adeli. He always focused ICAE on the right up-to-date topics. For instance, neural networks were already the centre of some articles in the first year of appearance 


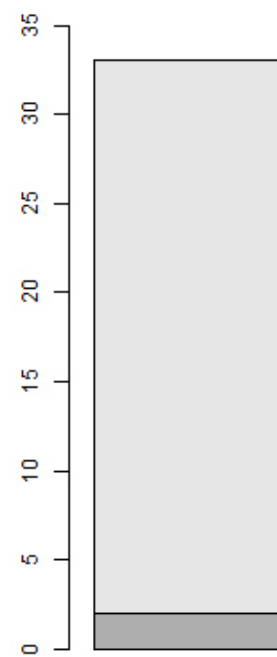

1993

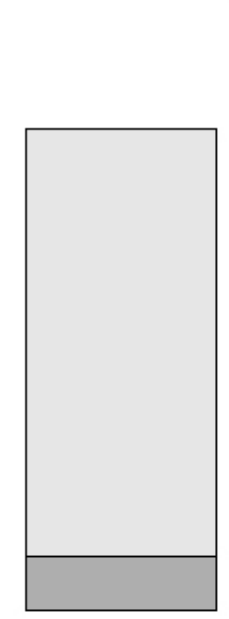

2000 $\square$ Neural networks

Deep learning

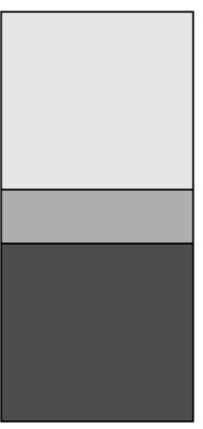

2020

\section{$\square$ Other}

Fig. 2. Proportion of ICAE papers with a focus on neural networks.

of the journal. At that time, neural networks played only a minor role in engineering, but ICAE published already pioneering papers in this field. In the following years, there was a slow but continuous increase of the proportion of papers involving neural networks. And as Fig. 2 demonstrates, in 2020 around half of the papers in ICAE had a strong focus on neural network. And the large majority of these papers were devoted to deep learning approaches, a topic that only started to dominate the field of neural networks just during the last decade.

And finally, from my personal experience of submitting a paper to ICAE, I can say that the reviewing process was rigorous, but very constructive. I received 6 reviews, more than any other journals I have published in. We obtained feedback from the reviewers that enabled us to publish finally a paper with ICAE considerably improved compared to the original contribution. We also got strong constructive support and advice from Prof. Adeli. And as a reviewer, I have experienced that he is determined to collect the reviews in time. Few journals have such a renowned Editor-in-Chief with six honorary doctorates, an h-index of more than 100 and many scientific achievements and awards that cannot be listed within the limited space of an editorial. There are journals with an electronic submission system with area editors to take care of the review process where the Editor-in-Chief lends his name to the journal, but has otherwise very limited duties. Not so with ICAE where Prof. Adeli guarantees personally for the quality of the journal.

What remains is to congratulate ICAE to its coming $30^{\text {th }}$ anniversary and Professor Adeli to the impressive success of the journal. I look forward to the further development of this excellent journal.

\section{Frank Klawonn}

Head of Biostatistics

Helmholtz Centre for Infection Research,

Braunschweig, Germany

E-mail: frank.klawonn@helmholtz-hzi.de

$\&$

Department of Computer Science

Ostfalia University, Wolfenbüttel, Germany

E-mail: f.klawonn@ostfalia.de 